\title{
Análise Temporal da Incidência de Leishmaniose Tegumentar em Municípios da Região do Parque Estadual do Aguapeí - SP
}

\author{
Temporal Analysis of the Incidence of Cutaneous Leishmaniasis in the Cities of \\ Aguapeí State Park Region - São Paulo State
}

\begin{abstract}
Análisis Temporal de la Incidencia de Leishmaniasis Tegumentaria en Municipios de la Región del Parque Estadual del Aguapeí - SP
\end{abstract}

Natália Poiani Henriques ${ }^{1}$

\begin{abstract}
RESUMO: A Leishmaniose Tegumentar (LT) é uma doença infecto-parasitária, não contagiosa, transmitida aos mamíferos através da picada de flebotomíneos, infectados por protozoários do gênero Leishmania. Registrada em todas as regiões brasileiras desde 2003, esta doença apresenta incidência variável e diferentes perfis epidemiológicos. O presente estudo analisou temporalmente a incidência da LT na população dos municípios que integram o Parque Estadual do Aguapeí (PEA), São Paulo, Brasil. Para tanto, acessou-se o número de casos humanos confirmados de LT, por município de infecção, de 1998 a 2016, disponíveis no Centro de Vigilância Epidemiológica, da Secretaria de Saúde do Estado e dados na literatura. O registro da doença na área de estudo remonta a década de 1930 e dados recentes indicam a transmissão da LT em intensidade variável. No ano de 2015 a intensidade de transmissão da LT foi considerada alta nos municípios de São João do Pau D'Alho e Nova Independência e média em Junqueirópolis e Monte Castelo. Conclui-se que o Parque está inserido em municípios com histórico de incidência da doença, sendo importante o incentivo à realização de monitoramento de tendências espaço-temporais e de estudos sobre as condições relacionadas à transmissão da LT nesta região.
\end{abstract}

PALAVRAS-CHAVE: Leishmaniose Tegumentar. Incidência. Parque Estadual do Aguapeí.

\begin{abstract}
Cutaneous Leishmaniasis $(C L)$ is a non-contagious infectious-parasitic disease transmitted to mammals through the bite of a sandflies infected by protozoa of Leishmania genus. It has been registered all over Brazil since 2003. This disease shows variable incidence and different epidemiological profiles. The present paper made a temporal analysis about the CL incidence over the population that integrates the municipalities of Aguapei State Park in São Paulo State, Brazil. For this purpose, it was accessed the number of CL confirmed cases in humans between 1998 and 2016 that were available at the Epidemic Surveillance Center of State Secretary of Health and literature data. The disease register of the area of study comes from 1930s and current data indicates CL transmission in variable intensity. In 2015 the intensity of CL transmission was considered high level in

\footnotetext{
${ }^{1}$ Graduada em Ciências Biológicas, Mestre em Geografia, Faculdade de Ciências e Tecnologia da Universidade Estadual Paulista, campus Presidente Prudente. Parque Estadual do Aguapeí - Fundação para a Conservação e a Produção Florestal do Estado de São Paulo. Endereço: Rua São Salvador, n. 983, Centro, CEP 17890-000, Junqueirópolis - SP. E-mail: natihenriques@yahoo.com.br.
} 
Análise Temporal da Incidência de Leishmaniose Tegumentar em Municípios da Região do Parque...

the towns of São João do Pau D'Alho and Nova Independência and medium level in Junqueirópolis and Monte Castelo. It is possible to conclude that the Park is among the cities where there is register of this illness. For this reason, it is important to encourage the monitoring of space-time trends and studies about the conditions related to CL transmission in this region.

KEYWORDS: Cutaneous Leishmaniasis. Incidence. Aguapeí State Park.

RESUMEN: La Leishmaniasis Tegumentaria (LT) es una enfermedad infecto-parasitaria, no contagiosa, transmitida a los mamíferos a través de la picadura de flebotomos, infectados por protozoarios del género Leishmania. Registrada en todas las regiones brasileras desde 2003, esta enfermedad presenta incidencia variable y diferentes perfiles epidemiológicos. El presente estudio analizó temporalmente la incidencia de la $L T$ en la población de los municipios que integran el Parque Estadual del Aguapeí (PEA), São Paulo, Brasil. Para esto, se accedió al número de casos humanos confirmados de LT, por municipio de infección, de 1998 a 2016, disponibles en el Centro de Vigilancia Epidemiológica, de la Secretaria de Salud del Estado y datos en la literatura. El registro de la enfermedad en el área de estudio remonta a la década del 30 y datos recientes indican la transmisión de la $L T$ en intensidad variable. En el año de 2015 la intensidad de transmisión de la $L T$ fue considerada alta en los municipios de São João do Pau D'Alho e Nova Independencia y media en Junqueirópolis y Monte Castelo. Se concluye que el Parque está dentro de municipios con historia de incidencia de la enfermedad, siendo importante el incentivo a la realización de monitoreo de tendencias espacio-temporales y de estudios sobre las condiciones relacionadas a la transmisión de la $L T$ en esta región.

PALABRAS-CLAVE: Leishmaniasis Tegumentaria. Incidencia. Parque Estadual del Aguapeí.

\section{INTRODUÇÃO}

A Leishmaniose Tegumentar (LT), também conhecida como Leishmaniose Cutânea, é uma doença infecto-parasitária, não contagiosa, transmitida aos mamíferos através da picada de fêmeas do inseto conhecido por flebotomíneo, infectadas por protozoários do gênero Leishmania (BRASIL, 2017; CENTERS OF DISEASE CONTROL AND PREVENTION, 2015; WORLD HEALTH ORGANIZATION, 2016). A LT é caracterizada por úlcera típica em áreas expostas da pele, geralmente indolor, variável em tamanho, número e aparência, sendo que o agravamento de sua condição clínica pode vir a produzir deformidades no ser humano (BRASIL, 2017; CENTERS OF DISEASE CONTROL AND PREVENTION, 2015).

Registrada nas Américas, Europa, África e Ásia, estima-se que de 0,7 a 1,2 milhões de novos casos da doença ocorram a cada ano em todo o mundo (CENTERS OF DISEASE CONTROL AND PREVENTION, 2015; WORLD HEALTH ORGANIZATION, 2016), estando - Brasil entre os dez países que juntos respondem por aproximadamente $75 \%$ dos casos mundiais e apresentam as maiores incidências em todo o mundo (WORLD HEALTH ORGANIZATION, 2016). 
$\mathrm{Na}$ América Latina a doença é endêmica em 18 países, apresentando-se em diferentes intensidades de transmissão (ORGANIZAÇÃO PAN-AMERICANA DA SAÚDE, 2017). A classificação da intensidade de transmissão da doença é possível a partir do cálculo do Indice Composto da Leishmaniose Tegumentar (ICLT) podendo ser considerada baixa, média, alta, intensa ou muito intensa. Proposto pela Organização Pan-Americana da Saúde, o ICLT é formado por três indicadores: número de casos novos, taxa de incidência de casos e densidade de casos, e as categorias geradas podem direcionar e priorizar as ações de vigilância, prevenção e controle (BRASIL, 2017).

Esta doença vem sendo registrada em todas as regiões brasileiras desde $2003 \mathrm{com}$ incidência variável. Em 2015, a maior incidência foi registrada nos estados da região Norte e a menor nos estados da região Sul (BRASIL, 2017). No país, a doença ocorre em ambos os sexos e em todas as faixas etárias, sendo que no ano de 2015 predominaram os casos em maiores de 10 anos (92,1\%), no sexo masculino (73\%), 57\% deles em homens acima de 20 anos (ORGANIZAÇÃO PAN-AMERICANA DA SAÚDE, 2017).

Podendo ser considerada originalmente uma doença zoonótica, de ambiente florestal, sem a participação do homem no ciclo de transmissão (TOLEZANO, 1994), a doença apresenta atualmente três padrões epidemiológicos no país: o silvestre; o ocupacional e lazer; e o rural e periurbano (BRASIL, 2017).

No padrão silvestre a transmissão ocorre em área de vegetação primária e o ser humano é acometido ao entrar em contato com o ambiente silvestre; no padrão ocupacional e lazer a transmissão da doença está associada à exploração da floresta e derrubada de matas para construção de estradas, usinas hidrelétricas, instalação de povoados, extração de madeira, desenvolvimento de atividades agropecuárias, de treinamentos militares e ecoturismo, já no padrão rural e periurbano em áreas de colonização a transmissão da doença está associada ao processo migratório, ocupação de encostas e aglomerados em centros urbanos associados a matas residuais (BRASIL, 2017).

O perfil epidemiológico da transmissão da LT vem sendo modificado de acordo com o crescimento socioeconômico (CAMARGO-NEVES; BRASIL, 2003). A distribuição espacial da doença no país indica sua ampla dispersão, com forte concentração em algumas áreas, havendo áreas de menor endemicidade ou de ocorrência de casos isolados (COSTA, 2005). Os ciclos de transmissão variam conforme a região geográfica, apresentando diferenças quanto às espécies de parasitas, vetores e reservatórios envolvidos (RANGEL; LAINSON, 2003).

A distribuição geográfica da LT é influenciada por fatores climáticos, topográficos e pela vegetação, os quais, por sua vez, determinarão a distribuição da fauna flebotomínica, assim a distribuição das leishmanioses pode ser parcialmente explicada pela ampla distribuição dos vetores (CAMARGO-NEVES; CARVALHO, 2014; GOMES; ANTUNES, 
Análise Temporal da Incidência de Leishmaniose Tegumentar em Municípios da Região do Parque...

2002; RANGEL; COSTA). Costa (2005) considera ainda o risco da infecção humana em relação à história, economia e atividades sócio-políticas que determinam a forma de construção do espaço geográfico no qual as populações se instalam.

A área de estudo do presente trabalho compreende os seis municípios cuja parcela do território compõe o Parque Estadual do Aguapeí (PEA): Castilho, Guaraçaí, Junqueirópolis, Monte Castelo, Nova Independência e São João do Pau D'Alho, localizados na Bacia Hidrográfica do Rio Aguapeí, no oeste do estado de São Paulo. Neste contexto, o presente trabalho buscou analisar temporalmente a incidência da Leishmaniose Tegumentar na população dos municípios que integram o (PEA).

\section{A ÁREA DE ESTUDO}

A área de estudo está localizada na Bacia Hidrográfica do Rio Aguapeí, no oeste do estado de São Paulo e compreende seis municípios cuja parcela do território compõe o Parque Estadual do Aguapeí (PEA). Trata-se dos municípios de Castilho, Guaraçaí, Junqueirópolis, Monte Castelo, Nova Independência e São João do Pau D'Alho (figura 1) com população total equivalente a 54.398 habitantes (tabela 1).

Figura 1 - Mapa de localização da área de estudo

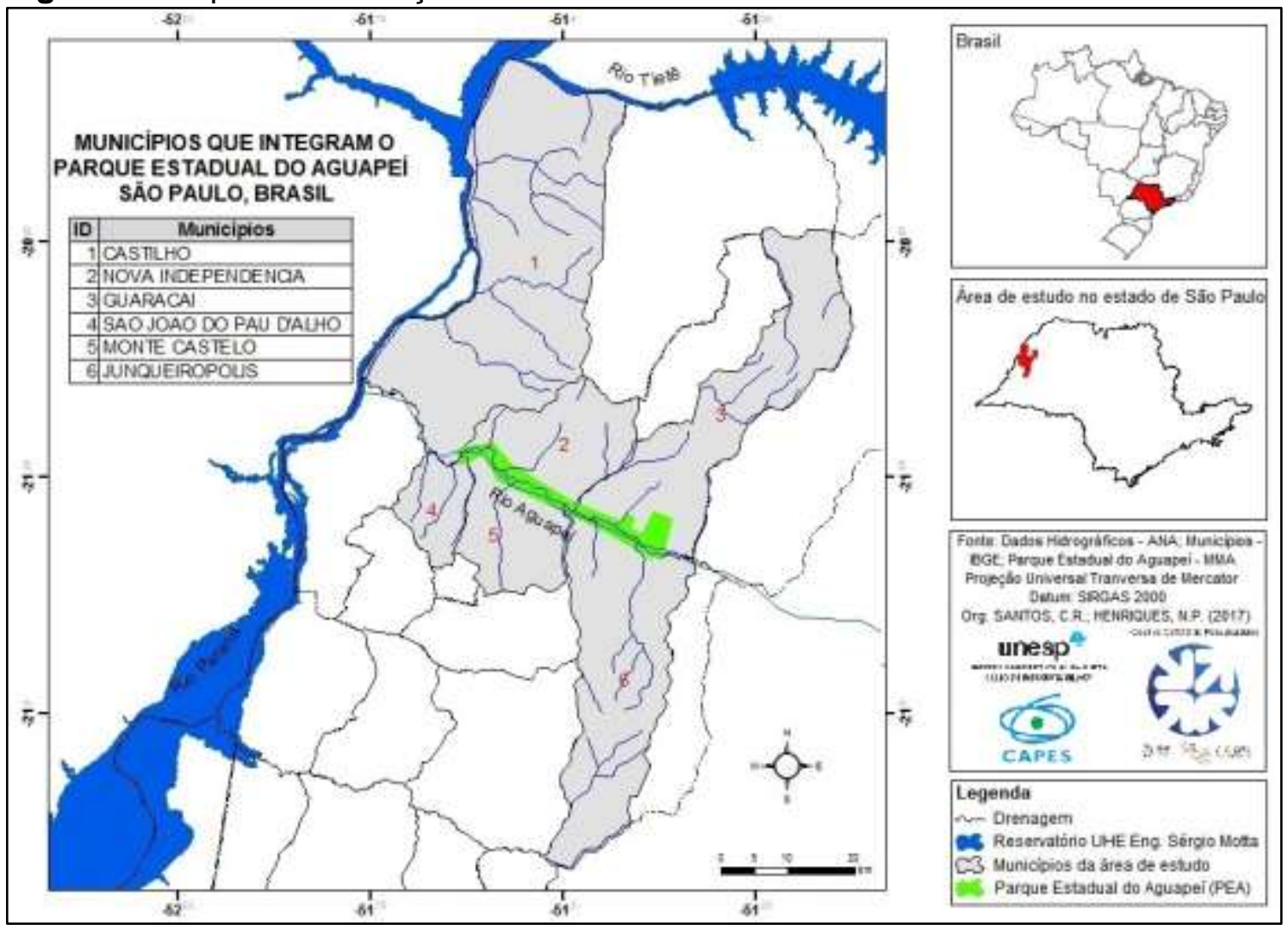

Fonte: adaptado de Henriques (2017). 
Tabela 1 - Dados geopopulacionais dos municípios da área de estudo

\begin{tabular}{|c|c|c|c|c|c|c|c|}
\hline Município & $\begin{array}{l}\text { Área total } \\
\text { do } \\
\text { município } \\
\text { (ha) }\end{array}$ & $\begin{array}{l}\text { IÁrea do } \\
\text { município } \\
\text { na UC } \\
\text { (ha) }\end{array}$ & $\begin{array}{l}\text { Distância } \\
\text { entre área } \\
\text { urbana e a } \\
\mathrm{UC}^{\mathrm{c}}(\mathrm{km})\end{array}$ & $\begin{array}{l}\text { Populaçã } \\
\text { o total }^{\text {d }}\end{array}$ & $\begin{array}{l}\text { Pop. } \\
\text { Urbana }^{d}\end{array}$ & $\begin{array}{l}\text { Pop. } \\
\text { Rural }^{d}\end{array}$ & $\begin{array}{l}\text { Densidade } \\
\text { demográfica } \\
\left({\left.\text { hab } / \mathbf{k m}^{2}\right)^{\mathrm{d}}}\right.\end{array}$ \\
\hline Castilho & 106.580 & 42,58 & 30 & 18.003 & 13.586 & 4.417 & 16,89 \\
\hline Guaraçaí & 56.987 & $3.092,43$ & 26 & 8.435 & 6.654 & 1.781 & 14,80 \\
\hline Junqueirópolis & 58.296 & $1.670,52$ & 23 & 18.726 & 15.399 & 3.327 & 32,12 \\
\hline Monte Castelo & 23.257 & $2.177,57$ & 10 & 4.063 & 3.211 & 852 & 17,47 \\
\hline Nova Independência & 26.578 & $2.014,46$ & 10 & 3.068 & 2.446 & 622 & 11,54 \\
\hline S. J. do Pau D'Alho & 11.772 & 46,39 & 12 & 2.103 & 1.705 & 398 & 17,86 \\
\hline Total & 283.470 & $9.043,95$ & - & 54.398 & 43.001 & 11.397 & - \\
\hline
\end{tabular}

Fonte: Organizado pela autora a partir de: Fundação SEADE (2010), São Paulo (2010) e IBGE (2010). Nota: a- FUNDAÇÃO SEADE (2010); b-SÃO PAULO (2010); c - Menor transecto traçado entre os limites da área urbana e o limite da UC-imagem do Google Earth; d-IBGE (2010).

Inserida no Bioma de Mata Atlântica, em área originalmente coberta por Floresta Estacional Semidecidual (FES), a cobertura vegetal original desta região foi devastada no início do século passado no processo de sua colonização, sendo que a história da ocupação e uso do solo do oeste paulista esteve relacionada à expansão da cultura cafeeira e da ferrovia, que levou à sua colonização no início do século XX (BOIN, 2000). Nas últimas décadas a região tem passado por novas transformações quanto ao uso do solo, sendo expressiva a conversão das pastagens em cultivos de cana-de-açúcar (SÃO PAULO, 2010).

Nesta região o clima é tropical quente e úmido (com chuvas de verão), e com um a dois meses de estação seca (inverno). A precipitação média anual é de 1.250 milimetros e a temperatura média anual superior a $18^{\circ} \mathrm{C}$, sendo janeiro o mês mais chuvoso (com precipitação média de 200 milimetros) e julho o mês mais seco é julho (com precipitação média de 25 milimetros) (SÃO PAULO, 2010).

A criação de uma Unidade de Conservação (UC) de proteção integral no cenário regional foi essencial à proteção de parte dos varjões que resistiram ao processo de ocupação do estado de São Paulo e à conservação da biodiversidade local associada a este tipo de vegetação. O PEA foi criado como forma de compensação ambiental pelos impactos causados pela construção da Usina Hidrelétrica Engenheiro Sérgio Motta no ano de 1998 (Decreto Estadual no 43.269, de 02 de julho de 1998) e possui uma área de 9.043,970 hectares, o equivalente a 3,2\% da área de estudo (SÃO PAULO, 2010).

Administrado atualmente pela Fundação para Conservação e Produção Florestal do estado de São Paulo, o PEA possui regularização fundiária e limites definidos, plano de manejo aprovado, sede implantada e está aberto para visitação. 


\section{PROCEDIMENTOS METODOLÓGICOS}

A taxa de incidência é um indicador epidemiológico que se refere ao número de casos novos confirmados de uma determinada doença, por 100 mil habitantes, na população residente em determinado espaço geográfico, no ano considerado (ANDRADE; CAMPOS, 2001; REDE INTERAGENCIAL DE INFORMAÇÃO PARA A SAÚDE, 2008; SOARES). Assim, a taxa de incidência estima o risco de ocorrência de LT numa determinada população em intervalo de tempo determinado, e a população exposta ao risco de adquirir a doença, estando relacionada à exposição de indivíduos a picadas de fêmeas de flebotomíneos infectadas com protozoários do gênero Leishmania (REDE INTERAGENCIAL DE INFORMAÇÃO PARA A SAÚDE, 2008).

Na presente análise, calculou-se a taxa de incidência da doença a partir do número de casos humanos confirmados de LT, por município de infecção, no período de 1998 a 2016, disponíveis na base de dados do Centro de Vigilância Epidemiológica "Prof. Alexandre Vranjac", da Secretaria de Saúde do Estado de São Paulo (CVE-SES-SP) e da base populacional do IBGE (2017), considerando as estimativas populacionais para cada ano de notificação. O método de cálculo baseia-se no resultado do número de casos novos de LT notificados na população residente dividido pela população total residente no período determinado, multiplicado posteriormente por 100.000 .

Para a análise dos resultados considerou-se os conceitos básicos para a investigação relacionados à ocorrência de surto, onde: surto refere-se à ocorrência epidêmica, em que os casos estão relacionados entre si, em uma área geográfica pequena e delimitada, como bairros, distritos ou em uma população institucionalizada ou restrita; e ocorrência de surtos refere-se à presença de casos de LT em uma área sem transmissão/silenciosa ou aumento de casos em relação ao número esperado em áreas com transmissão. (BRASIL, 2017).

Em relação à classificação dos municípios quanto à transmissão da doença, os mesmos foram considerados como municípios sem transmissão ou silencioso - aqueles municípios sem registro de ocorrência de casos humanos autóctones de LT nos últimos três anos - ou municípios com transmissão - aqueles municípios com histórico de registro de casos humanos autóctones de LT, contínuos ou não, nos últimos três anos. (BRASIL, 2017).

Consideraram-se, ainda, os estudos da literatura relacionados à ocorrência da doença na área de interesse e o estágio atual de implantação do Parque Estadual do Aguapeí, consultando-se a gestão da Unidade de Conservação a respeito das formas de interação entre a sociedade e a natureza que ocorrem nestes espaços especialmente protegidos. 


\section{A INCIDÊNCIA DA LEISHMANIOSE TEGUMENTAR NOS MUNICÍPIOS DA ÁREA DE ESTUDO}

Os resultados demonstraram que o número de novos casos humanos confirmados de LT, por município de infecção, no conjunto dos seis municípios da área de estudo, totalizou 68 casos no período de 1998 a 2016, o equivalente a 0,68\% dos casos notificados em todo o território paulista no mesmo período (9.912 casos).

Observou-se que as notificações de 1998 a 2014 estiveram entre 0,14 e 0,71\% dos casos notificados em todo estado de São Paulo e que no ano de 2015 houve um incremento significativo na participação nos casos totais registrados no estado e a área de estudo foi responsável por $8,85 \%$ dos casos estaduais, conforme figura 2 . Tal incremento sinaliza para uma situação epidemiológica que requer atenção e monitoramento de tendências posto que poderia estar refletindo mudanças regionais (RANGEL; COSTA; CARVALHO, 2014).

Figura 2 - Participação da área de estudo nos casos totais de Leishmaniose Tegumentar notificados no estado de São Paulo, de 1998-2016

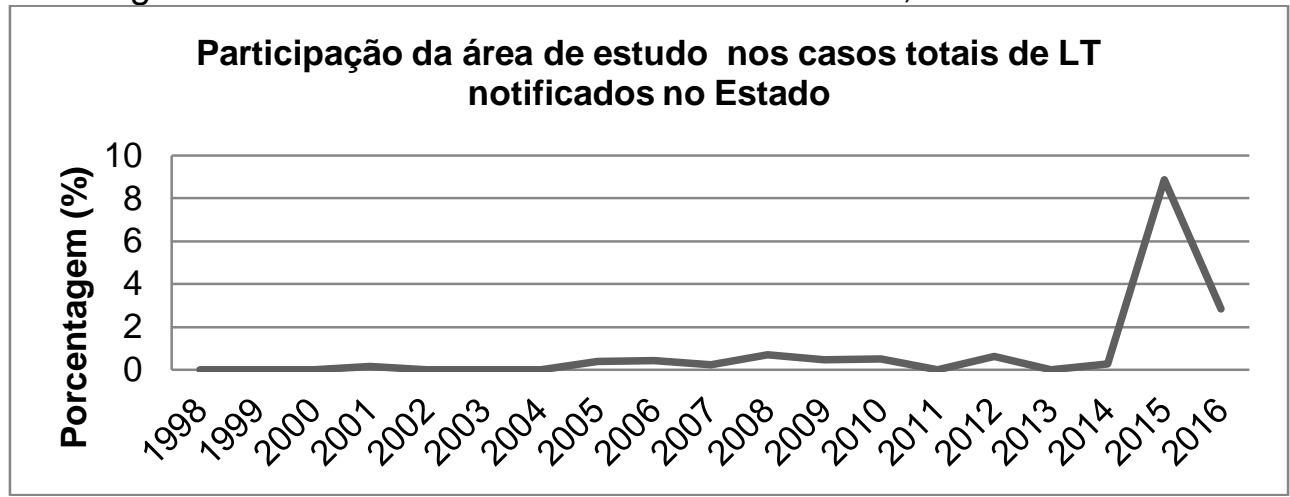

Fonte: São Paulo (2017).

Ao tratar das mudanças ambientais e a disseminação da doença no país, Rangel, Costa e Carvalho (2014) incluem em suas conclusões a importância da realização de estudos que visem à caracterização e o monitoramento de tendências espaço-temporais da doença, uma vez que tais estudos auxiliam na identificação de áreas de riscos (receptivas) para novos surtos e grupos de população em maior risco de infecção, levando a proposição de ações dirigidas ao controle da doença mais eficientes.

No período analisado, as taxas de incidência da doença na população residente nos municípios estudados oscilaram entre 4,95 e 233,54 por 100.000 habitantes (tabela 2). A utilização deste indicador epidemiológico permitiu uma análise da situação considerando a população exposta de cada localidade e possibilitando a comparação dos municípios entre si e os dados analisados indicam que a região apresenta condições que asseguram a 
Análise Temporal da Incidência de Leishmaniose Tegumentar em Municípios da Região do Parque...

manutenção do ciclo de transmissão da LT e que a população residente esteve exposta aos vetores.

Tabela 2 - Taxas de incidência de Leishmaniose Tegumentar (por 100 mil habitantes), por município, de 1998 a 2016

\begin{tabular}{l|cccccc}
\hline Ano & Castilho & Guaraçaí Junqueirópolis & $\begin{array}{c}\text { Monte } \\
\text { Castelo }\end{array}$ & Nova Independência & $\begin{array}{c}\text { São João do Pau } \\
\text { D’Alho }\end{array}$ \\
\hline 1998 & - & - & - & - & - & - \\
1999 & - & - & - & - & - & - \\
2000 & - & - & - & - & - & - \\
2001 & - & - & 5,91 & - & - & - \\
2002 & - & - & 5,93 & - & - & - \\
2003 & - & - & - & - & - & - \\
2004 & - & - & - & - & - & - \\
2005 & - & - & 6,04 & 27,06 & - & - \\
2006 & - & - & - & - & - & 116,89 \\
2007 & - & - & - & - & - & 46,90 \\
2008 & 6,23 & - & 5,07 & 24,21 & - & - \\
2009 & - & - & 5,01 & 24,21 & 36,47 & 45,64 \\
2010 & - & - & 5,34 & - & 65,19 & - \\
2011 & - & - & - & - & - & - \\
2012 & - & - & 10,53 & - & - & - \\
2013 & - & - & - & - & - & - \\
2014 & - & 11,69 & - & - & & 233,54 \\
2015 & 5,03 & 46,92 & 79,74 & 167,14 & 167,22 & 93,63 \\
2016 & 9,94 & 11,77 & 4,95 & 71,62 & - & \\
\hline
\end{tabular}

Fonte: São Paulo (2017).

No ano de 2015, a média da taxa de incidência da LT nas Américas foi de 18,35 casos por 100.000 habitantes, destacando-se a alta incidência registrada em Suriname onde foram registrados 218,48 casos por 100.000 habitantes (ORGANIZAÇÃO PAN-AMERICANA DA SAÚDE, 2017). Em 2015, 46.082 casos da doença foram notificados em 17 países da América Latina, 19.395 deles concentrados no Brasil, dos quais 18.324 casos caracterizavam-se pela forma cutânea da doença no país (ORGANIZAÇÃO PANAMERICANA DA SAÚDE, 2017).

Neste mesmo ano, nos municípios estudados, verificou-se um incremento significativo da incidência da doença no ano de 2015 nos municípios de São João do Pau D'Alho $(233,54)$, Nova Independência $(167,22)$ e Monte Castelo $(167,14)$, sugerindo a ocorrência de um surto nos mesmos. Em 2015, a classificação epidemiológica dos municípios quanto à intensidade da doença indicou para uma transmissão baixa em Castilho e Guaraçaí, média em Junqueirópolis e Monte Castelo e alta em Nova Independência e São João do Pau D' Alho (ORGANIZAÇÃO PAN-AMERICANA DA SAÚDE, 2017). 
A transmissão da doença em intensidade variável nos municípios da área de estudo sinaliza para a necessidade de acompanhamento contínuo das taxas de incidências da população em questão e dos aspectos eco-epidemiológicos local.

Antes mesmo do ano de 1998 havia no estado de São Paulo registros de casos humanos de LT e a ocorrência de grandes surtos da doença data do final do século XIX e início do século XX (TOLEZANO, 1994; TOLEZANO et al., 2001). As atividades humanas, decorrente da abertura de novas estradas ferroviárias, que envolviam a extração de produtos naturais e a expansão das áreas cafeeiras, modificaram a paisagem do estado de São Paulo, e, ao mesmo tempo, trouxeram a mudança epidemiológica deste agravo (TOLEZANO, 1994).

Naquele período os casos apresentavam o perfil ocupacional da doença, associados às atividades laborais em áreas de desmatamentos (TOLEZANO, 1994; TOLEZANO et al., 2001). Tolezano (1994) relata, com base na bibliografia, que na região noroeste do estado de São Paulo, a doença se espalhou de Bauru e Araçatuba, estendendo-se para a região de Sorocaba e mais tarde, durante a década de 1930, na região de Marília e Tupã. No período de 1935 e 1939, a Região de Saúde em que estão inseridos os municípios da área de estudo representava $91,6 \%$ dos casos estaduais da doença, de acordo com Tolezano (1994).

No início da década de 1950, Forattini (1954) desenvolveu estudos sobre a biologia dos vetores da LT na região da bacia do rio Paraná, época marcada por forte transformação da paisagem resultante da ocupação da porção oeste do estado. As observações feitas de 1951-1953 demonstraram a ocorrência de casos de LT na região dos municípios da área de estudo, 30 deles registrados em uma fazenda situada nos municípios de Junqueirópolis e Pacaembu, conhecida como Fazenda Santa Maria do Aguapeí. Estes dados possibilitam o reconhecimento da ocorrência da doença na área de interesse não sendo possível inferir sobre sua taxa de incidência neste período.

As atividades laborais neste contexto estavam centradas na derrubada das matas e na implantação das primeiras lavouras do Oeste Paulista, o que aproximava o homem de uma condição favorável ao adoecimento e, assim, a região da Alta Paulista e Alta Sorocabana despontavam como zona de alta endemicidade de LT no estado (FORATTINI, 1954; TOLEZANO, 1994).

$\mathrm{Na}$ sequência, observou-se no cenário estadual uma redução das notificações da doença que parecia estar relacionada ao grau de devastação da cobertura vegetal nativa (CAMARGO-NEVES; GOMES; ANTUNES, 2002; TOLEZANO, 1994). Entre 1962 e 1973, houve relatos de casos da doença nas áreas rurais das cidades localizadas em florestas residuais, bem como na região da capital paulista, sendo que estudos nas décadas 
Análise Temporal da Incidência de Leishmaniose Tegumentar em Municípios da Região do Parque...

subsequentes levaram ao reconhecimento de uma nova característica epidemiológica da doença onde a transmissão ocorria no domicílio e no peridomicílio (TOLEZANO, 1994).

A partir de 1980, os casos humanos de LT passaram a ser notificados no estado de São Paulo associados aos fatores antrópicos, uma transmissão que não estava restrita aos ambientes florestados caracterizando, assim, uma transição no perfil epidemiológico da LT (CAMARGO-NEVES; GOMES; ANTUNES, 2002). A epidemiologia da LT no estado passou a ser caracterizada por micro-surtos e casos esporádicos, com grande número de focos distribuídos em todas as regiões paulistas (TOLEZANO, 1994; TOLEZANO et al., 2001). Nos anos de 2001 e 2002, Camargo-Neves e Brasil (2003), constatam que a transmissão da LT ocorreu de forma esporádica, corroborando com estudos anteriores de Gomes e Camargo-Neves (1998) e Camargo-Neves (1999).

Gomes e Camargo-Neves (1998) e Camargo-Neves (1999) referem-se ainda à ocorrência de surtos epidêmicos em algumas regiões paulistas, associados ao processo de produção do espaço geográfico, seja pela expansão urbana em áreas próximas de mata, seja pela ocupação do solo por novas áreas de plantio.

Para a identificação dos fatores ambientais e sociais determinantes na incidência da LT na área de interesse, sobretudo de modo a reconhecer os aspectos relacionados ao aumento da incidência observada nos anos de 2015, sugere-se a continuidade de estudos visando à constatação de correlação entre a incidência da doença e variáveis de natureza sociodemográficas, climáticas e geográficas, uma vez que estas duas últimas estão fortemente relacionadas às flutuações nas populações dos vetores da LT (SILVA; LATORRE; GALATI, 2010).

Estudos futuros poderiam adotar modelos múltiplos de regressão tais como empregados por Silva, Latorre e Galati (2010) na análise da incidência da LT na região do Vale do Ribeira/SP. Dentre as variáveis de natureza sociodemográficas, as autoras consideraram a população média, população rural e urbana média, taxa de analfabetismo, índice de desenvolvimento humano, crescimento geométrico, densidade demográfica, escolaridade e renda per capita.

Em relação às condições climáticas, Silva, Latorre e Galati (2010) consideraram a temperatura do ar (máxima, média e mínima) e a chuva acumulada (máxima e por ano). Já em relação às variáveis político-geográficas e físico-biológicas, consideraram a existência de loteamentos, distritos industriais e domicílios em área rural, domicílios urbanos e grau de urbanização, existência de cortiços e favelas, existência de unidades de conservação e de parque temático em área rural, existência de Conselho Municipal de Meio Ambiente, altitude e registro de flebotomíneos. No referido estudo, as variáveis analisadas apontaram na direção da urbanização da doença. 
Em relação às variáveis que poderiam estar relacionadas à incidência da doença nos municípios que integram a área do PEA, o que se pode afirmar até o presente momento é o registro de flebotomíneos em Castilho, Guaraçaí, Junqueirópolis, Nova Independência e São João do Pau D'Alho (HENRIQUES, 2017).

\section{A NOÇÃO DE RISCO EM SAÚDE}

Considerando que constantemente os indivíduos estão submetidos ao risco de doenças e agravos, a análise do risco é importante, sobretudo, por possibilitar o estabelecimento de medidas de proteção, mitigação e eliminação do mesmo (LIMA, 2016). Antes, cabe destacar que risco é um conceito polissêmico, de entendimento variável ao longo do tempo, adotado nas mais diversas áreas (GONDIM, 2007; LIMA, 2016). Para Gondim (2007) o risco, em sua concepção moderna, tem como ideia central o controle do futuro, surgindo em oposição ao conceito de fatalidade e destino, entendendo-se que toda atividade humana possui um risco associado, o qual pode ser reduzido evitando-se determinadas atividades, ainda que não seja possível eliminá-lo por completo.

Ao tratar das ameaças à saúde (health hazard) e riscos à saúde (health risk), Gondim (2007, p. 90) diferencia estes dois conceitos, de modo que a 'ameaça' seja entendida como "[...] um objeto ou um conjunto de situações que podem potencialmente trazer dano à saúde de uma pessoa" e que o 'risco' seja entendido como "[...] uma probabilidade de que uma pessoa sofrerá um dano devido a uma ameaça em particular."

No raciocínio epidemiológico, Almeida Filho (2000, p. 10) esclarece que as doenças são entendidas como "[...] eventos ou episódios, mensuráveis por meio de probabilidades condicionantes de ocorrência", ou seja, nem todas as pessoas que experimentam ou se expõem a uma situação de risco irão adoecer, mas que tem maiores chances que outras que se encontra em situação diferente (NATAL, 2004). Assim, ressalta-se que o conceito de risco e a noção de incerteza estão intimamente relacionados e que, no cotidiano dos indivíduos, certos riscos são aceitos e outros são evitáveis, e que em ambos os casos a noção de dano faz-se presente (LIEBER; ROMANO-LIEBER, 2003).

Para Gondim (2007) cada medida tomada no sentido de promover e proteger a saúde das pessoas e os ambientes vai depender da severidade do dano e do grau de incerteza em torno do problema. Deste modo, o conhecimento acerca do problema é fundamental para a proposição de intervenções que possam minimizá-lo, agregando-se, para tanto, a ciência e a percepção dos indivíduos expostos ao risco. No que se refere às leishmanisoes, a detecção de uma única espécie de flebotomíneos de importância epidemiológica já configura fator de risco para a doença (CAMARGO-NEVES, 2004). 
Análise Temporal da Incidência de Leishmaniose Tegumentar em Municípios da Região do Parque...

Sem a pretensão de estabelecer fatores e ambientes de risco e condições para transmissão da LT nos municípios da área de estudo, propõe-se a seguir uma reflexão sobre a implantação de uma unidade de conservação no contexto regional e a preocupação relacionada à nova dinâmica local, evidenciada pela ampliação das formas de interação da sociedade e da natureza, sejam elas ocupacionais ou de ecoturismo e lazer, em seu interior e entorno.

\section{A IMPLANTAÇÃO DO PARQUE ESTADUAL DO AGUAPEÍ NO CONTEXTO REGIONAL}

Nas últimas duas décadas, têm sido expressiva a conversão de pastagens em cultivos de cana-de-açúcar nos municípios da área de estudo, no mesmo período em que se observaram articulações e esforços visando à implantação do PEA no cenário regional (SÃO PAULO, 2010). O PEA (figura 3) se enquadra entre as UCs do grupo de proteção integral onde se prioriza a manutenção dos ecossistemas livres de alterações causadas por interferência humana, permitindo-se a utilização indireta de seus recursos, sendo proibido o consumo, coleta, dano ou destruição dos recursos naturais (BRASIL, 2000), sendo que esta área protegida pela UC representa apenas 3,2\% da área de estudo.

Figura 3 - Imagens aéreas do Parque Estadual do Aguapeí
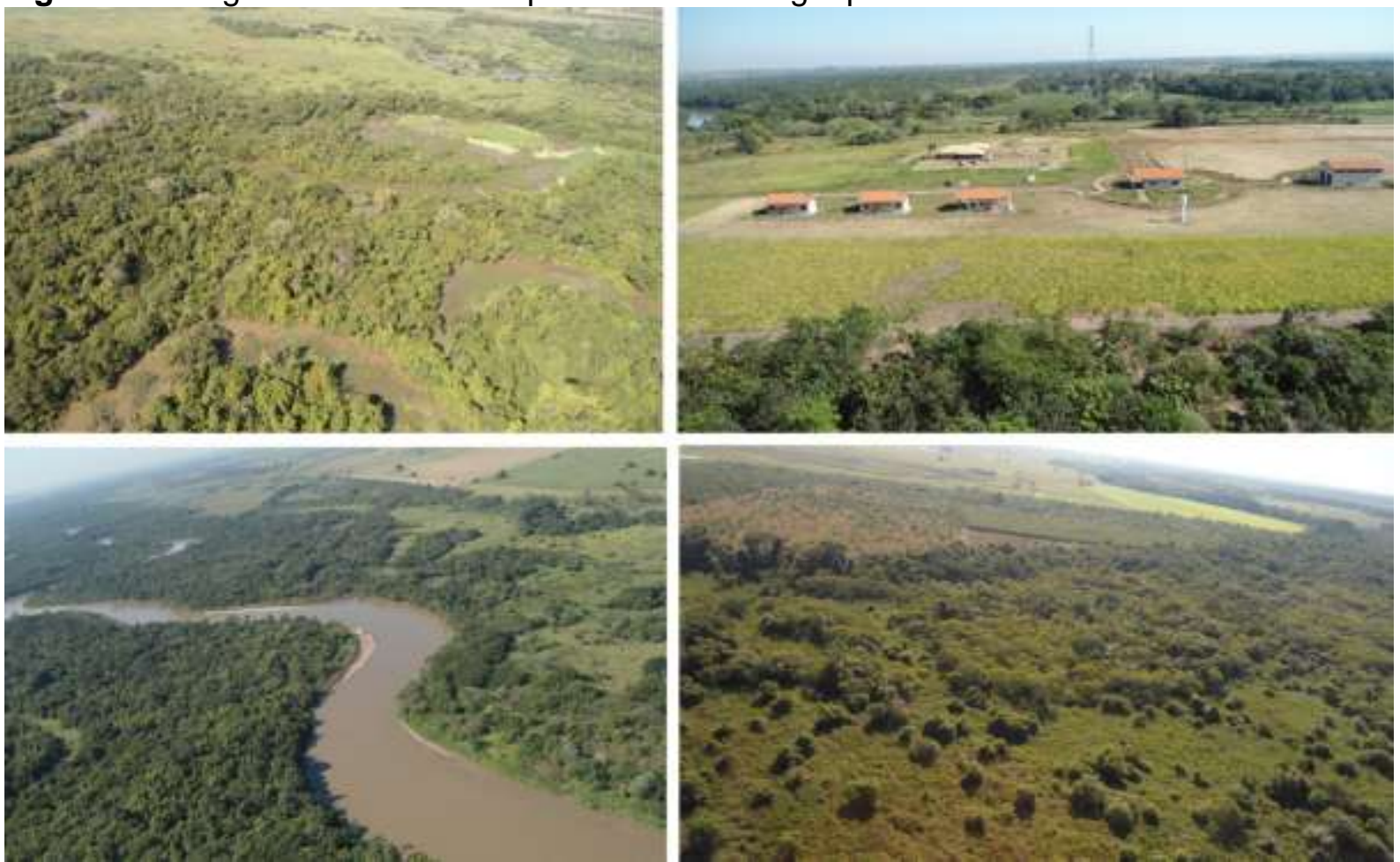

Fonte: Arquivo de fotos da autora (2016). Imagens áreas do Parque Estadual do Aguapeí. No canto superior, à direita, encontram-se, na zona de uso intensivo, a sede administrativa e a infraestrutura destinada ao uso público. 
De modo geral, em parques são incentivadas as atividades de pesquisa científica, estudos e monitoramento ambiental, sendo permitido o desenvolvimento de atividades de educação e interpretação ambiental, recreação e turismo ecológico (BRASIL, 2000). Assim, o processo de implantação desta categoria de UC compreende uma diversidade de formas de interação entre a sociedade e a natureza, tanto de caráter ocupacional quanto de ecoturismo e lazer, compreendendo atividades administrativas, de manejo, proteção, pesquisa e visitação pública, interações que devem ser estabelecidas de forma planejada, considerando as normas e zoneamento de cada UC (BRASIL, 2000; SÃO PAULO, 2014).

Com a criação do PEA no cenário regional, uma série de normativas legais passou a vigorar neste território que está sob um regime especial de administração, destacando-se, neste contexto, a proibição da pesca nos recursos hídricos presentes na UC e as restrições em relação à sua ocupação e ao adensamento demográfico em seu entorno (SÃO PAULO, 2010; 2014).

De acordo com São Paulo (2010), a pesca é proibida no interior do parque enquadrando-se como crime ambiental e, apesar disso, trata-se de uma atividade que, assim como a caça, ameaçam a proteção da biodiversidade existente na UC. No que tange à transmissão da LT, a permanência humana junto às matas ciliares para a prática da pesca tem sido uma das formas de interação entre a sociedade e a natureza relacionada à transmissão da doença, tal como apontado nos estudos de Castro et al. (2002) e Bocchi (2007). A esse respeito, entende-se que as restrições de cunho protetivo relacionadas à caça e pesca no interior do PEA podem contribuir para a diminuição da exposição humana às condições para transmissão desta doença.

Rangel (2004, p. 54) pondera de que "[...] não é possível assegurar que em todos os fragmentos de matas poupadas à destruição resultaram condições naturais para ocorrência do caso humano da LT", ainda que doença esteja associada a quase todas as áreas de floresta residual e a quase todos os rios que atravessavam o Estado de São Paulo, tal como apontado por Tolezano (1994).

$\mathrm{Na}$ presente análise, não foi identificada na literatura consultada a existência de estudos locais visando à comprovação de condições que assegurem a manutenção do ciclo da LT na área protegida pelo PEA, à exceção de Henriques (2017) que constatou a existência de espécies de flebotomíneos de interesse epidemiológico em determinadas áreas do PEA. Já no entorno não imediato da UC, Odorizzi e Galati (2007) constataram a alta densidade de uma espécie vetora da LT em rancho de pesca no município de Mirandópolis, que pode estar associada à transmissão da doença na região.

Outro aspecto associado ao caráter protetivo desta UC que também poderia contribuir para a diminuição da exposição humana às condições para transmissão desta doença está relacionado à restrição quanto à ocupação no interior da UC e ao adensamento demográfico 
Análise Temporal da Incidência de Leishmaniose Tegumentar em Municípios da Região do Parque...

no entorno imediato ao PEA. A relação entre os processos de organização e ocupação do espaço e a LT tem sido tratada por diversos autores, tais como os estudos realizados por Kawa e Sabroza (2002) e Costa (2005).

Os diferentes perfis epidemiológicos que a LT apresenta atualmente junto ao histórico de sua incidência na população dos municípios que integram o PEA validam a preocupação relacionada à implantação desta UC, ainda que não tenha sido estudada a existência de correlação espacial entre os casos registrados da doença e a área protegida pelo parque e seu entorno. Como exposto inicialmente, tal preocupação reside no processo de implantação da UC, marcado por uma dinâmica peculiar que compreende a alocação de recursos humanos necessários ao manejo e proteção do patrimônio natural, em áreas próximas de áreas florestadas ou mesmo em seu interior, onde também ocorre a interação sociedade e natureza através das atividades de uso público e ecoturismo.

Com base no estágio atual da implantação do PEA, verificada junto à gestão da UC, das relações socioespaciais de caráter ocupacional que ocorrem no interior da UC, entendese que os indivíduos mais expostos às condições de transmissão da LT são aqueles que convivem habitualmente e desenvolvem suas relações no ambiente de trabalho próximo às matas, ou em seu interior, em períodos compatíveis com os hábitos dos vetores da doença. De acordo com Brazil e Brazil (2003), os vetores da LT apresentam hábitos predominantemente crepusculares e noturnos, com pico de atividade alimentar variando conforme a espécie, havendo poucas espécies de hábitos diurnos, estando estas em locais com pouca luminosidade.

Assim, os serviços de vigilância patrimonial, concentrados nas imediações das edificações da sede administrativa e prestados de forma ininterrupta, e de modo semelhante as periódicas incursões na mata para fiscalização ambiental, despontam como as relações socioespaciais de caráter ocupacional que merecem atenção no contexto da implantação da UC assim como as eventuais pesquisas científicas que exigem um contato direto com as áreas florestadas e a permanência em ambiente propício a picadas de insetos em período compatível com os hábitos dos flebotomíneos.

Concomitantemente, a transmissão da LT associada a atividades de lazer em reservas florestais ou próximo a matas também foi abordada em diversos estudos, tais como os desenvolvidos por Castro et al. (2002) e Bocchi (2007), configurando o ecoturismo como uma das atividades historicamente associada à transmissão desta doença (BRASIL, 2017).

Observou-se que as atividades de lazer, ecoturismo e uso público em desenvolvimento no PEA têm sido planejadas e realizadas em período diurno, o que diminuiria a exposição dos indivíduos à condição de transmissão tendo em vista os hábitos dos vetores da LT. Verificou-se ainda que no atual estágio de implantação do Parque não há 
área de camping disponível aos visitantes e eventuais pernoites ocorrem em alojamentos localizados na zona de uso intensivo, condição que pode diminuir a exposição noturna a picadas de insetos dadas à existência de telagem nas janelas dos alojamentos.

Fato é que as espeficidades dos ambientes deveriam ser levadas em consideração no planejamento e execução das mais diversas ações. Assim, enquanto não são desenvolvidos estudos sobre as reais condições que possam assegurar, ou não, o ciclo da LT no interior do PEA, cabe ressaltar a adoção de medidas preventivas de ambientes individuais ou coletivos, relacionadas à transmissão da doença, quais sejam: uso de repelentes durante a exposição a ambientes onde os vetores habitualmente possam ser encontrados; evitar a exposição nos horários de atividades do vetor (crepúsculo e noite); uso de mosquiteiros de malha fina e telagem de portas e janelas das edificações; manejo ambiental por meio de limpeza da área de uso intensivo da UC, a fim de alterar as condições do meio que propiciem o estabelecimento de criadouros do vetor; e o destino adequado do lixo orgânico produzido na UC, a fim de impedir a aproximação de mamíferos comensais prováveis fontes de infecção para os flebotomíneos (BRASIL, 2017).

Tais medidas preventivas são igualmente aplicáveis às relações estabelecidas no entorno do Parque, as quais se podem acrescer: a adoção de faixa de segurança entre as residências e as matas (em áreas potenciais de transmissão), a manutenção de animais domésticos distantes do intradomicílio durante a noite e a limpeza periódica de seus abrigos (BRASIL, 2017).

Pela presente reflexão entende-se que a noção de risco em saúde e a compreensão das múltiplas dimensões envolvidas no processo saúde-doença e dos fatores relacionados à ocorrência e incidência da LT são essenciais para a não-estigmatização destes espaços especialmente protegidos como fonte de doenças.

\section{CONCLUSÕES}

A partir da presente análise verificou-se a ocorrência de casos humanos de Leishmaniose Tegumentar na região onde está inserido o Parque Estadual do Aguapeí nas décadas de 1930 e 1950, época em que os casos estiveram associados ao perfil ocupacional da doença em um contexto histórico marcado pela ocupação da região.

Nos últimos 20 anos a doença tem sido caracterizada na área de estudo pela ocorrência de casos esporádicos e micro surtos, havendo atualmente transmissão em intensidade variável nos seis municípios estudados. Assim, faz-se necessária a realização de monitoramento de tendências espaço-temporais e de estudos sobre as condições e riscos associados à transmissão da LT nesta região. 
Análise Temporal da Incidência de Leishmaniose Tegumentar em Municípios da Região do Parque...

Dada a existência de uma UC no cenário regional, as restrições de cunho protetivo relacionadas à proibição da caça e pesca no interior do Parque poderiam contribuir para a diminuição da exposição humana às condições para transmissão da doença assim como as restrições relacionadas à ocupação humana e o adensamento demográfico em seu entorno.

A implantação da UC possibilita, por outro lado, a interação sociedade-natureza tanto em caráter ocupacional quanto de ecoturismo e lazer, assim enquanto não se reconhece a existência de condições que assegurem a manutenção do ciclo da LT no interior do Parque e os riscos associados à transmissão da doença, a adoção de medidas de cunho preventivo é essencial.

\section{REFERÊNCIAS}

ALMEIDA FILHO, N. O conceito de saúde: ponto-cego da epidemiologia? Revista Brasileira de Epidemiologia, São Paulo, v. 3, n. 1-3, p. 4-20, 2000.

BOCCHI, M. R. Transmissão de Leishmaniose Tegumentar Americana na Região de São José do Rio Preto. 2007. Dissertação (Mestrado em Ciências da Saúde) - Faculdade de Medicina de São José do Rio Preto - FAMERP, São José do Rio Preto, 2007.

BOIN, M. N. Chuvas e erosões no oeste Paulista: uma análise climatológica aplicada. Tese (Doutorado em Geociências) - Universidade Estadual Paulista, Rio Claro, 2000.

BRASIL. Lei 9.985 de 20 de julho de 2000. Regulamenta o art. 255, §1ํㅡ, incisos I, II, III e VII da Constituição Federal, institui o Sistema Nacional de Unidades de Conservação da Natureza e dá outras providências, Brasília, 2000.

Ministério da Saúde. Secretaria de Vigilância em Saúde. Manual de vigilância da leishmaniose tegumentar. Brasília, DF: Ministério da Saúde, 2017.

BRAZIL, R. P.; BRAZIL, B. G. Bionomia. In: RANGEL, E. F.; LAINSON, R. (Org.).

Flebotomíneos do Brasil. Rio de Janeiro: Fiocruz, 2003. p. 257-274.

CAMARGO-NEVES, V. L. F. Aspectos epidemiológicos e avaliação das medidas de controle da leishmaniose visceral americana no Estado de São Paulo, Brasil. 2004. Tese (Doutorado) - Faculdade de Saúde Pública Universidade de São Paulo. São Paulo, 2004.

Características da transmissão da Leishmaniose Tegumentar Americana no Estado de São Paulo, Brasil. 1999. Dissertação (Mestrado em Saúde Pública) - Faculdade de Saúde Pública da Universidade de São Paulo. São Paulo, 1999.

; BRASIL, M. T. L. R. F. Leishmaniose Tegumentar Americana no Estado de São Paulo: situação epidemiológica 2001 - 2002. Revista da Sociedade Brasileira de Medicina Tropical, Uberaba, MG, v. 36, p. 30-35, 2003.

.; GOMES, A. C.; ANTUNES, J. L. F. Correlação da presença de espécies de flebotomíneos (Diptera: Psychodidae) registros de casos da leishmaniose tegumentar americana no Estado de São Paulo, Brasil. Revista da Sociedade Brasileira de Medicina Tropical, Uberaba, MG, v. 35, n. 4, p. 299-306, jul-ago. 2002.

CASTRO, E. A.; SOCCOL, V. T.; MEMBRIVE, N.; LUZ, E. Estudo das características epidemiológicas e clínicas de 332 casos de leishmaniose tegumentar notificados na região norte do estado do Paraná de 1993 a 1998. Revista da Sociedade Brasileira de Medicina Tropical, Uberaba, MG, v. 35, n. 5, p. 445-52. 2002. 
CENTERS OF DISEASE CONTROL AND PREVENTION - CDC. 2015. ParasitesLeishmaniasis. Biology. Disponível em:

<http://www.cdc.gov/parasites/leishmaniasis/biology.html>. Acesso em: 18 jan. 2016.

COSTA, J. M. L. Epidemiologia das Leishmanioses no Brasil. Gazeta Médica da Bahia, Salvador, v. 75, n. 1, p. 3-17, 2005.

FORATTINI, O. P. Algumas observações sobre biologia dos flebotomos (Diptera: Psychodidae) em região da bacia do rio Paraná (Brasil). Arquivos da Faculdade de Higiene de São Paulo, São Paulo, v. 8, p. 15-136, 1954.

FUNDAÇÃO SEAD. 2010. Perfil dos municípios paulista. Disponível em: <http://www.perfil.seade.gov.br/>. Acesso em: 10 jun. 2015.

GOMES, A. C.; CAMARGO-NEVES, V. L. F. Estratégias e perspectivas de controle da Leishmaniose Tegumentar Americana no Estado de São Paulo. Revista da Sociedade Brasileira de Medicina Tropical, Uberaba, MG, v. 31, n. 6, p. 553-558, 1998.

GONDIM, G. M. M. Do Conceito de risco ao da precaução: entre determinismos e incertezas. In: FONSECA, A. F.; CORBO, A. M. D. (Org.). O território e o processo saúdedoença. Rio de Janeiro: EPSJV/Fiocruz, 2007. p. 87-120.

HENRIQUES, N. P. Leishmaniose Tegumentar Americana e a relação doença-espaço geográfico: aportes para a gestão dos Parques Estaduais do Aguapeí e do Rio do PeixeSP. Dissertação (Mestrado em Geografia) - Faculdade de Ciência e Tecnologia da Universidade Estadual Paulista, Presidente Prudente, 2017.

IBGE. Censo demográfico 2010. Disponível em: <http://www.cidades.ibge.gov.br>. Acesso em: 15 jul. 2017.

Estimativas da população residente com data de referência 1 de julho de 2017. Ago. 2017. Disponível em: <https://biblioteca.ibge.gov.br/visualizacao/livros/liv100923.pdf>. Acesso em: 15 jul. 2017.

KAWA, H.; SABROZA, P. C. Espacialização da leishmaniose tegumentar na cidade do Rio de Janeiro. Cadernos de Saúde Pública, Rio de Janeiro, v. 18, n. 3, p. 853-865, maio/jun. 2002.

LIEBER, R. R.; ROMANO-LIEBER, N. S. Risco, incerteza e as possibilidades de ação na saúde ambiental. Revista Brasileira de Epidemiologia, São Paulo, v. 6, n. 2, p. 121-134, 2003.

LIMA, S. C. Território e promoção da saúde: perspectivas para a atenção primária à Saúde. Jundiaí: Paco Editorial: 2016.

NATAL, D. Fundamentos da Saúde Pública. In: PHILIPPI JUNIOR., A.; ROMÉRO, M. A.; BRUNA, G. C. (Ed.). Curso de gestão ambiental. Barueri, SP: Manole, 2004. p. 333-374.

ODORIZZI, R. M. F. N.; GALATI, E. A. B. Flebotomíneos de várzea do rio Aguapeí, região noroeste do Estado de São Paulo, Brasil. Revista de Saúde Pública, São Paulo, v. 41, n. 4, p. 645-52, 2007.

ORGANIZAÇÃO PAN-AMERICANA DA SAÚDE - OPAS. Leishmaniose Visceral; América do Sul. Washington, 2017. (Leishmanioses: informe epidemiológico das Américas. n. 5, abr. 2017). Disponível em: <http://iris.paho.org/xmlui/handle/123456789/34113>. Acesso em: 7 jul. 2017.

RANGEL, E. F.; COSTA, S. M. CARVALHO, B. M. Environmental Changes and the geographic spreading of American cutaneous Leishmaniasis in Brazil. In: CLABORN, D. Leishmaniasis: trends in epidemiology, diagnosis and treatment. London: InTech Ed. Cap. 1. 2014. p. 3-25. Disponível em: http://dx.doi.org/10.5772/5720. Acesso em: 27 dez. 2016. 
Análise Temporal da Incidência de Leishmaniose Tegumentar em Municípios da Região do Parque...

.; LAINSON, R. Ecologia das leishmanioses. In: RANGEL, E. F.; LAINSON, R. (Org.).

Flebotomíneos do Brasil. Rio de Janeiro: Fiocruz, 2003. p. 291-309.

RANGEL, O. Estudo da expansão das áreas geográficas de transmissão de

Leishmaniose Tegumentar Americana (LTA) na região de Campinas Estado de São

Paulo Brasil. Tese (Doutorado em Saúde Coletiva) - Faculdade de Ciências Médicas da

Universidade Estadual de Campinas, Campinas, 2004.

REDE INTERAGENCIAL DE INFORMAÇÃO PARA A SAÚDE. Indicadores básicos para a saúde no Brasil: conceitos e aplicações. Brasília: Organização Pan-Americana da Saúde, 2008. Disponível em: <http://tabnet.datasus.gov.br/tabdata/livroidb/2ed/indicadores.pdf>. Acesso em: 15 jan. 2017.

SÃO PAULO. Secretaria de Estado da Saúde. Leishmaniose Tegumentar Americana casos confirmados segundo GVE de infecção e ano de diagnóstico Estado de São

Paulo: período de 2007 a 2018. Disponível em: <http://www.saude.sp.gov.br/cve-centro-devigilancia-epidemiologica-prof.-alexandre-vranjac/areas-de-vigilancia/doencas-detransmissao-por-vetores-e-zoonoses/agravos/leishmaniose-tegumentar-americana/dadosestatisticos>. Acesso em: 25 jul. 2017.

Secretaria de Meio Ambiente. Fundação Florestal. Instituto Florestal. Plano de Manejo: Parque Estadual do Aguapeí. São Paulo, 2010. Disponível em:

<http://fflorestal.sp.gov.br/planos-de-manejo/planos-de-manejo-planos-concluidos/>. Acesso em: 7 out. 2014.

Secretaria de Meio Ambiente. Manual de gestão das unidades de conservação do Estado de São Paulo. São Paulo, 2014.

SILVA, A.; LATORRE, M. R. D. O.; GALATI, E. A. B. Fatores relacionados à ocorrência de leishmaniose tegumentar no Vale do Ribeira. Revista da Sociedade Brasileira de Medicina Tropical, Uberaba, MG, v. 43, n. 1, p. 46-51, jan. /fev., 2010.

SOARES, D. A.; ANDRADE, S. M.; CAMPOS, J. J. B. Epidemiologia e Indicadores da Saúde. In: ANDRADE, S. M.; SOARES, D. A. (Org.). Bases da Saúde Coletiva, Londrina: UEL, 2001. p. 183-210.

TOLEZANO, J. E. Ecoepidemiological aspects of American Cutaneous Leishmaniasis in the state of são Paulo. Memórias do Instituto Oswaldo Cruz, Rio de Janeiro, v. 89, p. 427-34, 1994.

. et al. Epidemiologia da Leishmaniose Tegumentar Americana (LTA) no Estado de São Paulo III: influência da ação antrópica na sucessão vetorial da LTA. Revista do Instituto Adolfo Lutz, São Paulo, v. 60, n. 1, p. 47-51, 2001.

Recebido: agosto de 2018. Aceito: dezembro de 2018. 